\title{
Computational Models of Affect and Fuzzy Logic
}

\author{
Albert van der Heide ${ }^{1}$ Daniel Sánchez ${ }^{1}$ Gracian Trivino $^{1}$ \\ ${ }^{1}$ European Centre for Soft Computing \\ Mieres, Asturias, Spain \\ \{albert.vdheide, daniel.sanchezf, gracian.trivino\}@softcomputing.es
}

\begin{abstract}
In light of recent research and empirical findings a new view on emotion and affect has surfaced. Within this view 'feeling' (core affect) is separated from 'emotion' (psychological construction). This model appears to be a good candidate for a computation model of affect and emotion. We argue that fuzzy logic is the perfect tool for its implementation, for the modeling of core affect dynamics, the linguistic description of emotions, the description of a cognitive rule base, as well as the mapping between the core affect level and the cognitive level.
\end{abstract}

Keywords: Fuzzy Logic, Affective Computing, Emotion

\section{Introduction}

In recent years researchers have become more aware and recipient to the importance of affect and emotion in human cognition, which can be seen in works such as $[1,2]$. Because of this research it has become evident that emotion is fundamental to human experience, influencing cognition, perception, and everyday tasks such as learning, communication, and even rational decision-making. However, emotion has been largely ignored in computational systems, and as a result have often created a frustrating experience for people. For a large part this negligence has been due to the misunderstanding of affect and emotion, as well as the difficulty of measuring it. As a consequence of the rise and interest in this new view on human cognition, as well as the strong push from the field of Human Computer Interaction to create empathic humanlike interaction, there is a growing interest in the subject of affect and emotion modeling.

Affective Computing [3] is a field in which emotions and other affective phenomena are studied and incorporated into computing. Through the study and design of new systems that do incorporate the affective component, new technologies are developed that better address human needs. There are two general objectives that can be identified in the field. First there are investigators which try to model and simulate human reasoning processes [4] with the objective to reproduce human behavior. A second group of researchers are interested in Human
Computer Interaction, and for these researchers the underlying neurological or psychological processes are of less importance, as long as the applications can give the impression to be humanized in interaction.

In this paper we introduce our view of fuzzy logic as a suitable tool that can contribute in several different ways to the development of computational affective models and systems. For this purpose, we elaborate on a relatively new view on affect and emotion proposed by Russell, named 'core affect' [5], what the consequences are of such a model for computational modeling of affect and emotion, and the important role fuzzy logic can play in this scenario. Russell's view on affect and emotion is the basis for the computational model we would like to advocate, and in previous publications we have experimented with the modeling of core affective phenomena $[6,7]$.

The paper is organized as follows: in section 2 we introduce the basic model we shall employ along the paper and the arguments posed by different authors to support this model. The two main levels of this model are described in sections 3 and 4 . In section 5 we discuss on the role fuzzy logic can play in the development of different components of computational models of emotion on the basis of the model introduced previously. Finally, in section 6 conclusions are given, as well as suggestions for future work.

\section{Models of emotion}

Computational models of emotion in the literature are inspired on theoretical, psychological models of emotion. Hence, a discussion about the latter is relevant before discussing on the former, particularly about the role fuzzy sets can play.

In many of the existing computational models, emotions are often seen as the result of 'appraisal processes'. In appraisal theories $[8,9]$ the relationship of the individual with the environment is expressed by means of appraisal rules that state how the individual (or agent) reacts emotionally to an event. In the process of determining the final emotion type two variables are of huge importance. One of these variables is related to a valence-like dimension, i.e. liking versus disliking, pleased versus displeased, love versus hate. A second impor- 
tant variable is the intensity of an emotion, which has a strong relation with the arousal of an agent, e.g. when an emotion type has a high intensity this would correlate with either very low or very high arousal. Various computational models use such appraisal processes as a cornerstone in their model, e.g. WASABI [10], FLAME [11], EMA [4], FearNot! [12], along with other variables that are considered of importance for cognition.

Appraisal rules appear to be an excellent way to define the relationship environment-agent. However, in human beings, these appraisals are insufficient to explain all of the complexity involved in the psychological phenomena of emotion. Hence, appraisal-based models are insufficient for providing computational models with a close human-like behavior. Beyond appraisals alone, humans experience 'feelings' without emotion, sometimes over longer periods of time, i.e. moods, and can have different personalities. All of these processes have an impact on appraisal as well as other processes in human beings. None of these elements are considered in appraisal-based models.

Another popular alternative for the representation of emotions and moods are dimensional models. There is much evidence supporting the idea that the emotions can be represented by means of an emotion space consisting of a limited number of dimensions [13]. In the particular case of [14], emotions are represented in a three-dimensional space in which the basic dimensions are labeled 'pleasure', 'arousal' and 'dominance' (often called PAD-space). Mehrabian showed empirically that each PAD component can be related to personality traits [15]. Many of these dimensional theories identify emotions with locations in PAD-space, i.e. emotion categories are identified with certain values in dimensional space. However, these dimensional categories have a number of limitations. As an example of such a limitation, the dimensional theories fail to explain how emotions such as fear, jealousy, shame and anger can be distinguished from one another.

Recently, psychologists started to investigate unconscious processes in emotions [16], concluding that there are levels of processing in 'affective functioning', and introducing a distinction between 'emotions' and 'feelings' to account for it [17]. According to these researchers, feelings are unconscious processes, roughly describing the brain's registration of body conditions and changes, whereas emotions are interpreted feelings, this interpretation process being cognitive and conscious [17].

In light of these new findings the currently used models seem inadequate and unable to account for the various phenomena that are of importance to and that influence affect and emotion. In [5, 18, 19], a new model based on the aforementioned recent studies is proposed. This model, sketched in figure 2, identifies two essential levels in the affective systems: on one level there is only a description in terms of feeling good or bad, energized or enervated, and on another level, the previous level as well as other processes are observed and explained in terms of emotion categories. The first level is called core affect, while the second level is related to conscious cognitive processes.

In sections 3 and 4 we will describe with more detail the two levels, core affect and cognition, as well as other elements in figure 2. Additionally, it should be noted that this view on affect and emotion has been applied and experimented upon in computational models of the authors, to which we refer in section 5 .

\section{Core affect}

The point of departure for the theory of core affect is that at the heart of emotion, mood, and any other emotionally charged event are states experienced as simply feeling good or bad, energized or enervated. These states are neuro physiological states which can be represented in a dimensional space. In section 3.1 we elaborate upon this representation scheme of core affect and how feelings are related. In section 3.2 the dynamics of core affect is discussed as well as some of the causal relations it has to other processes.

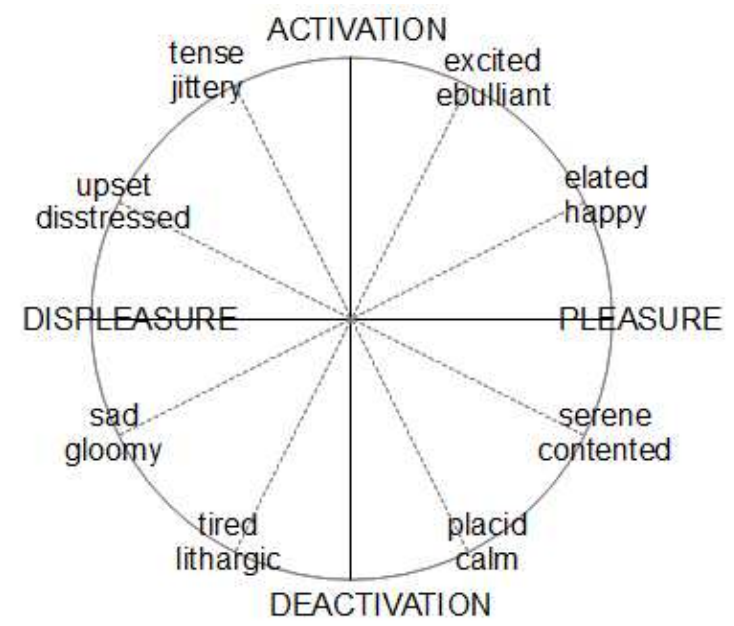

Figure 1: Prototypical feelings in core affect as suggested by Russell [5]. The labels in the figure do not represent emotions and are only indicative of sensation related to the particular area.

\subsection{Feelings and representation}

The modeling of core affect is based on a twodimensional space of pleasure/valence and arousal, which is similar to some dimensional theories. However, the areas in this space are related to sensations or feelings, e.g. the sensation of energized or enervated, of good or bad. This is contrary to conventional dimensional theories as they identify certain areas with certain emotions. In the two level view 


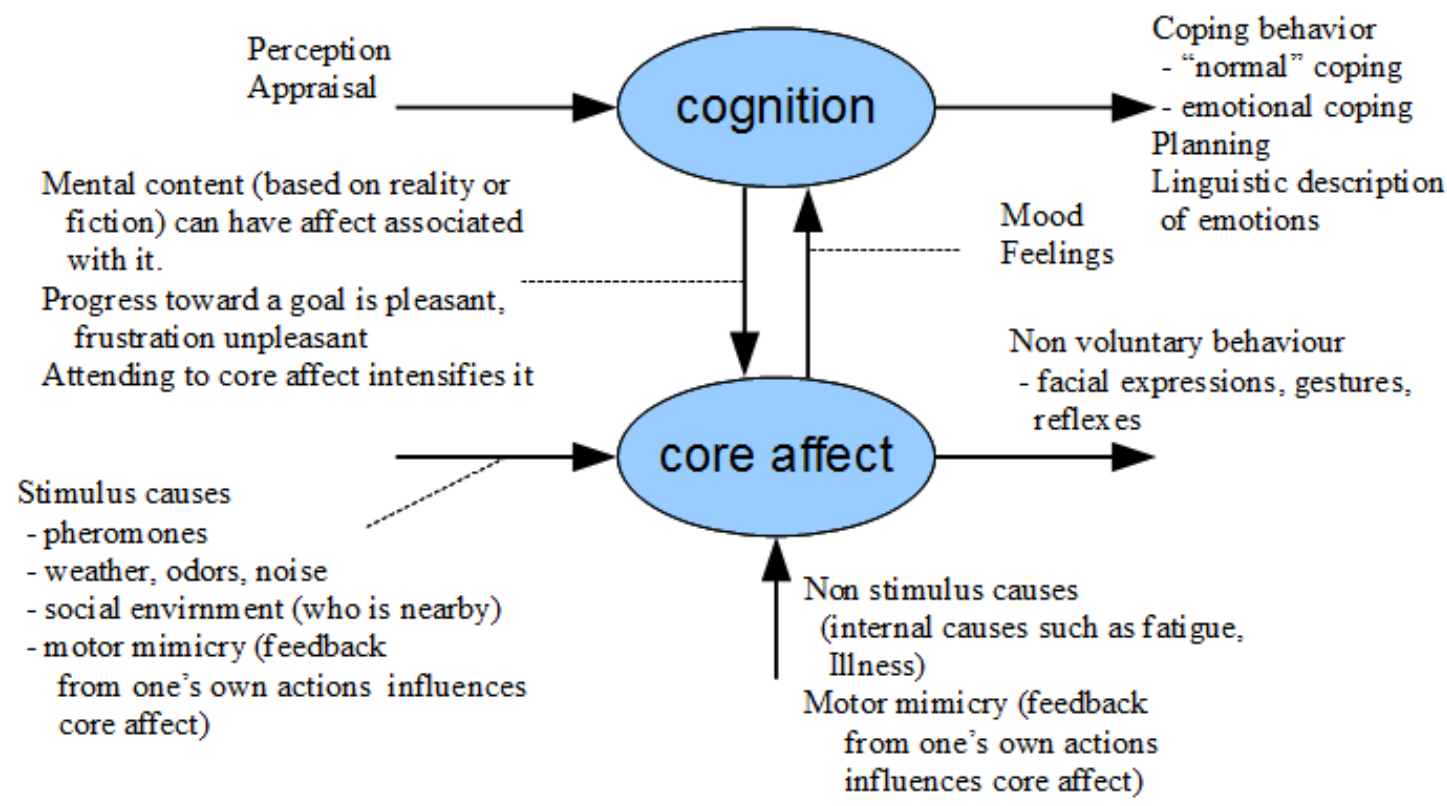

Figure 2: Core affect and the complexity of causal relations. On the 'left side' of the figure the environment influences are depicted, which internally are processed (center) and produce effects onto the external world on the 'right side' of the figure. This depiction is by no means exclusive of causal relations, only indicative of its complexity.

on emotion the sensations are but one of the necessary conditions for the experience of an emotion (see section 4).

The core affect space may be represented as a circle in a two dimensional space, as depicted in figure 1 (the labels in the figure do not represent emotions and are only indicative of sensations related to the particular area).

A person always has a core affect, which can be neutral (at the center point), moderate, or extreme (near outer circle). Extreme core affect is likely to be the focus of consciousness, while milder core affect usually is in consciousness' background. When there is a rapid change in core affect this will fill consciousness; when core affect is stable, even when not neutral, it tends to disappear from consciousness.

\subsection{Dynamics}

Core affect influences reflexes, perception, cognition, and behavior and is influenced by many internal and external causes. However, at the cognitive level the causes of the dynamics in 'core affect' are unknown. Quoting [5]:

A key to understanding core affect is that people have no direct access to these causal connections and limited ability to track this complex causal story. Instead, a person makes attributions and interpretations of core affect. Sometimes the cause is obvious, but at other times, one can undergo a change in core affect without knowing why.
The processes that influence the dynamics of core affect are not fully understood. However, there is evidence that there are genetically based individual differences related to average levels of core affect, its volatility, and its responsiveness to stimuli types, e.g. [20]. Additionally, people can seek to actively regulate their core affect by taking a morning coffee or the evening brandy. In addition more causal relations exist, which have been represented as arrows to and from core affect in figure 2 . In the next sections we will elaborate on cognition, in particular emotion, and how it relates to core affect.

\section{Cognitive experience}

The interrelation between cognition and core affect is broad, for example there is evidence for moodcongruent priming [21]; when feeling happy, like valenced information is recalled more easily. Additionally, when feeling happy one processes more positively the information about an object and overestimates its pleasantness. However, in this section we will focus our discussion on the relation between core affect and subset of cognitive processes, namely the experience of emotions.

\subsection{From core affect to conscious experience}

In the dimensional theories on emotion certain areas in dimensional space are related to or identified with a particular emotion type, e.g. basic emotions such as anger, disgust, fear, happiness, sadness, surprise. However, in the two level view on emotion 
this is different, as the same or overlapping areas in core affect can be related to multiple emotion types. In general the emotion categorization depends not only on core affect, but on additional variables or (cognitive) processes as well.

There is evidence in the literature that certain areas in dimensional space are related to basic emotions $[13,14,22,23]$ The two level view on emotion can account for this, as a core affect of pleasure may qualify as the emotion of happiness, a core affect of displeasure can count as a case of sadness. The combination of pleasure and high arousal may qualify as elation, the combination of displeasure and high arousal as anxiety. Core affect alone can cause basic emotions.

However, oftentimes the emotion categorization that takes place at the cognitive level is not only based upon core affect, but also upon additional non-affective processes (see section 4.2).

\subsubsection{Unattributed feelings}

Oftentimes in cognition a cause is identified and the sensation can be attributed to something, e.g. an object. It is in these situations that a cognitive categorization can be made, giving rise to an emotion. This categorization process is the subject of the next section.

In section 3.1 we briefly elaborated upon how certain areas of core affect are related to sensations. However, when core affect enters into consciousness, but it does not have any object it can be attributed to, then core affect is experienced as a feeling of being energized or enervated, feeling good or bad. According to Russell [5] we experience unattributed core affect as mood.

\subsection{Categorization}

In conventional models typically events trigger emotions which, in turn, can have different manifestations, e.g. a feeling, certain signals (facial expressions, etc), instrumental actions. Therefore in these models, an emotion is a mediator between a cause and various manifestations.

In the two level view emotion is the result of a cognitive psychological construction which determines the categorization [5]. This categorization is based upon a number of processes, some of which are cognitive, e.g. appraisal, perception of affective quality, emotional meta-experience, emotion regulation, while others are overt behaviors, e.g. action taken. We will not go into detail of all of these processes, as that would be out of the scope of this article. The interested reader is referred to [5] for an elaborate explanation. What is essential in this view is that 'core affect' is only one of the elements on which a categorization is based.

The categorization into an emotion is an after the fact account or explanation of the components involved, based upon similarity among aforementioned processes. None of the elements are caused by an event that could be called an emotion. Emotional episodes are each time constructed based on specific circumstances. In this view emotional episodes are not fixed by biological inheritance or human evolution (contrary to basic emotion theories) or fixed by social rules or categories (contrary to social constructionist theories).

\subsubsection{Folk psychology}

Contrary to the traditional view emotion categories are not well defined, there is no limited set of features that define it. However, there is a set of events that have resemblance among observed components and mental representations which cause a categorization. This set of events is picked out by an emotion label in language, e.g. fear or anger.

These emotion labels that denote the categories are part of common sense or folk psychology, i.e. are part of the set of assumptions, constructs, and convictions that make up the everyday language in which people discuss human psychology. Folk psychology includes everyday concepts such as 'beliefs', 'desires', 'fear', and 'hope'.

The process of the categorization into emotion is a cognitive process. The categorization, e.g. the 'felt' emotion, is a construction made by the person or agent based on core affect, of which the person can be aware or not, and many other elements in the situational context.

\subsubsection{Objects}

When an agent is experiencing a sensation and does not have an object to which it can be attributed, this can be experienced as mood, as pointed out in section 4.1.1. However, oftentimes cognition has identified a cause to which the sensation can be attributed. This cause could be, for example, another agent or the agent itself, resulting in different emotion categorizations. If the object to which the feeling is attritubed is another agent, the resulting categorization could be the emotion 'love', if the object is the agent itself the emotion category could be 'happy'.

The causal relation between core affect and its cause is unknown to the agent and cognition is constructing its explanation of phenomena. With this conceptual framework certain cognitive processes can be elegantly explained, for example misattribution [24]. In misattribution a change in core affect is due to a cause, but the change is misattributed at the cognitive level to something else, e.g. imagine the situation in which a person experiences a strong feeling of unpleasantness, due to his boss' punishment. Due to social norms the person cannot become angry with his boss. The person misattributes his feeling to a colleague, and becomes angry at his colleague. 
The view on emotions as psychological constructions deals well with problems that are traditionally present in dimensional theories, e.g. it can explain the distinction between the emotion categories anger and fear which in many dimensional theories map to similar areas in dimensional space [25], as the distinction between the two categories does not reside in an 'affective component' but depends upon a cognitive component. Additionally the core affect view on affect and emotion provides new possibilities and additional conceptual freedom, which potentially can account for a much larger part of the experimental data reported in the literature.

\subsection{From conscious experience to core affect}

Many processes can influence core affect. Among those processes are conscious cognitive processes. For example, core affect is especially subject to the drama taking place in consciousness, which can be based on reality (core affect change due to seeing a bear next to you), fiction (watching a scary movie), or imagination (imagining a sad situation). Planning and expectations are involved in affective phenomena as well, as progress towards a goal is associated with pleasant core affect while the obstruction of a goal can lead to frustration and unpleasant core affect. However, more commonly it is a series of events that is the cause of core affect, such as the cumulative stresses of a week on the job.

In computational models of affect and emotion these relations are usually materialized as coping strategies, e.g. [26, 27], which directly or indirectly (e.g. through appraisal of the changed environment) influence the affective state of the system.

In conclusion we can state that there is a high interrelation between the level of core affect and cognition. The identification of the interrelations is an ongoing subject of research. There is no doubt that core affect plays an important role in the categorization of emotions, and that certain areas in core affect are related to emotion types.

In the next section we will argue for the use of fuzzy logic to model the emotion categories that are present in natural language expressions, as emotions could be represented as linguistic variables.

\section{Fuzzy logic}

Fuzzy logic has been applied to many fields, from control theory to artificial intelligence. Fuzzy logic is an excellent tool when robust and approximate reasoning is required as opposed to exact and fragile. Variables in mathematics usually take numerical values, while in fuzzy logic applications the nonnumeric linguistic variables [28] are used. These linguistic variables facilitate the expression of rules and facts [29].

In everyday language and interaction no sharp concepts are used and the boundaries of concepts are vague. Fuzzy logic [30] provides a tool to model this vagueness. Furthermore fuzzy logic makes it possible to analyze and describe complex systems in linguistic terms instead of numerical values [31].

These advantages of fuzzy logic are of great value in affective computing, and more in particular, for use in computational models of affect and emotion, as we shall discuss in the next sections.

\subsection{Fuzzy Logic and Moods}

In section 3.1 the core affect space was presented and in section 4.1 we have elaborated upon the relation between core affect and the conscious experience of affect (sensations) and emotion. We have seen that the core affect space is related, in an unsharp fashion, to cognitive phenomena; linguistic labels can be roughly related to areas, as depicted in figure 1.

In section 4.1.1 it was argued how consciously experienced unattributed core affect can account for the phenomenon of mood, i.e. mood is free floating core affect. Certain moods have certain linguistic labels attached to them, e.g. people speak of being in a good mood or a bad mood. These labels denote folk psychological concepts that do not have sharp boundaries, but that are related to areas in the core affect space. Fuzzy logic is a perfect tool to bridge this semantic gap.

\subsection{Fuzzy Logic and Core Affect dynamics}

Moods are long lasting and have relatively stable core affect states related to them. This is contrary to the core affect related to emotions. Full blown emotional episodes have rather extreme dynamic changes in core affect related to them, e.g. changes in physiological body states such as high arousal or high negative valence. However, these rapid changes have a relatively short time frame. In addition to these dynamics, as seen in section 3.2 , there are interpersonal differences.

The dynamics of core affect are complex. A model of core affect should model the physiological changes in an agent. However, the dynamics of the system is usually described in folk psychological terms, i.e. linguistic terms related to emotions, moods, personalities or personality traits. Again there is a semantic gap that needs to be bridged.

A tool to model the dynamics of complex systems are Fuzzy Finite State Machines (FFSM) [32]. In previous studies, we have learned that FFSM are suitable tools for modeling systems that evolve in time following an approximately repetitive pattern $[32,33,34]$. Furthermore, in [35] we explored the possibilities of modeling the state of stress of a person using as sensors a skin conductivity meter and accelerometers, while in $[6,7]$ experiments have been conducted using FFSM's for emotion and personality simulation, in which the FFSM simulates 
the physiological processes. This conceptualization is similar to core affect.

These studies demonstrate the usefulness of FFSM's for the modeling of complex system dynamics using fuzzy rules. Furthermore, folk psychological concepts can be used as linguistic terms, which makes it possible to use a high descriptive level in the modeling of core affect dynamics, e.g. in terms of personality (quickly agitated, positive). As such the dynamics are defined by a set of rules using linguistic terms, which not only has the advantage of facilitating the understandability and interpretability of the system, but additionally many theories on personality use high level descriptions, e.g. [36]. For all of these reasons we propose FFSM's as an adequate tool to model the temporal dynamics of core affect.

\subsection{Fuzzy Logic and Emotions}

Not only linguistic concepts related to moods and personality are part of folk psychology (see section 4.2.1), but also the emotion categories. These common sense categories have unclear boundaries between them. To represent such vague affective categories found in language different approaches can be used of which fuzzy logic is a very good candidate [37]. Additionally, as with moods, these terms are related to fuzzy areas in core affect space. This fact is a strong argument to use fuzzy logic to model emotion categories [29]. However, additional cognitive processes are of importance to the categorization.

An example of an additional cognitive process is the attribution of core affect to an object (see section 4.2.2). The result of the attribution process could be of a crisp, statistical or fuzzy nature. As explained in section 4.2, the emotion categorization is a construction that is based on similarity among components. Or in the words of Russell [5]: The resemblance among components is a matter of degree. The borders between non-instances, instances, and prototypical instances are very fuzzy. Fuzzy logic can aggregate and fuse information from the all processes involved, being crisp, fuzzy or in between. Using this technique resemblance among various cognitive processes, as well as core affect, can be modeled.

\subsection{Fuzzy Logic and Cognition}

Cognition in humans is a highly complex process. All computational models of affect and emotion model this complexity to some extend. In various models there are internal representations of the outside world, of causal relations, of beliefs, desires and intentions, etc. The information on which cognition works can be of varying granularity, which is an important fact to take into account.

The large majority of computational systems on affect and emotion use rules to express cognitive functions such as appraisal and coping processes. These processes work on the fuzzy emotion concepts. Fuzzy concepts together with cognition which is expressed in the form of rules suggest the use of a fuzzy logic rule base system for the modeling of cognition. Actually, it has been shown in FLAME [11] that cognitive processes can be expressed perfectly in this way, as concepts as well as the appraisal rules were expressed using fuzzy logic and fuzzy rules respectively, to map events and expectations to emotional states and behaviors. Appraisal rules using these linguistic variables can be interpreted and understood in a very natural way. The authors note that fuzzy logic made it possible to achieve smooth transitions in the resultant behavior with a relatively small set of rules. However, FLAME's focus is on appraisal processes as well as learning, and does not incorporate the distinction between a core affect level and cognitive level.

Additionally, a problem which present day computational models do not deal with well is how to manage the multiple emotions. Commonly these models only allow one emotion to be active at any point in time, i.e. triggered emotions are filtered out. However, this is unnatural as it is possible for a human to experience (1) mixtures of emotions, and/or (2) conflicting emotions. Fuzzy Logic can provide an elegant way to model this fact, as it allows multiple emotions to be active to various degrees at any point in time.

\subsection{Fuzzy Logic and Integration of the System}

The domain of affect and emotion is currently being investigated in many fields, such as neurobiology, psychology, philosophy, artificial intelligence, etc. The phenomenon is complex and there are many ill-understood interdependencies among processes. In section 4.3 we elaborated upon the fact that cognitive processes can have an impact on core affect, for example perception of a smell or the memory of a loved one.

A large part of cognition in present day computational models is represented using high level information units, e.g. objects, facts, beliefs, intentions, etc. There is a relation between units at this cognitive level and the core affect space. However, this dependency of core affect on cognition is only one element of the system as a whole. In general computational systems of affect and emotions are designed for certain environments, which can be virtual or real. In these environments various inputs and outputs are dependent upon core affect (roughly unconscious and reflexive behavior) and cognition (roughly conscious and deliberative behavior).

Furthermore, the components involved in computational systems of affect and emotion have a huge semantical range. For example, one module can use representations in terms of Facial Action Coding 
System [38, 39], another module uses high level appraisal information, a third module uses raw data, while a fourth linguistic descriptions.

From a practical point of view one needs to take into account that new system modules are likely to be added as science advances in a particular field, introducing new representation schemes and/or changed interdependencies. This new information, which can vary from crisp to imprecise, has to be dealt with. A fuzzy logic representation at the heart of the system allow a relatively easy aggregation of this new knowledge as fuzzy logic is known for its ability to provide information fusion mechanisms, providing a bridge for existing semantic gaps. For all of these reasons we believe that fuzzy logic is an excellent tool for these particular kind of systems.

\section{Conclusions}

In this paper we have discussed the role fuzzy logic can play in affective computing. We have focused upon a new view on affect and emotion with proposes two levels in affective processing; a core affect level and a cognitive level, a view which is more in alignment with the current knowledge about the phenomena. We have argued for the important role fuzzy logic can play in the computational modeling of these processes. The two level view is the fundamental basis for the approach we use to model affect and emotion.

Core affect is the basic level on which all affect and emotion phenomena are dependent. The areas in core affect related to emotion categories are un-sharp. We proposed fuzzy logic as an excellent tool to model this mapping. We have argued that fuzzy logic, and in particular Fuzzy Finite State Machines, can be an appropriate tool to model the short, medium and long term dynamics of core affect.

Furthermore, affective experiences (feelings and emotions) are always a construction after the fact. Sometimes based upon core affect alone, but in general based upon a set of additional non affective processes. Furthermore, the experience of an emotion is a psychological construction, using the same categories found in folk-psychology. These everyday language categories are concepts that do not have well-defined sharp boundaries. It is for these domains that fuzzy logic was designed.

For a large part computational systems use rules to implement cognitive processes. These processes can also be performed using fuzzy rules and fuzzy concepts, as shown in FLAME [11]. An additional advantage is that the design of such systems is relatively easy since the rules use linguistic terms for emotion.

As future work we have foreseen the study of how fuzzy logic can be applied to model emotional meta experience, perception of affective quality, among others, as these processes also influence emotion construction. Nonetheless, further investigation into the specifics of the processes involved is necessary.

The implementation of a system simulating core affect dynamics is another theme for future work, in which feeling dynamics and how this relates to personality is of particular interest to us. First steps have been made into this direction by using FFSM's to model core affect dynamics in relation to personality [7] as well as stress [35].

\section{References}

[1] A.R. Damasio. Descartes' Error: Emotion, Reason, and the Human Brain. G.P. Putnam, New York, USA, 1994.

[2] A.R. Damasio. Looking for Spinoza: Joy, Sorrow, and the Feeling Brain. Harcourt Incorporated, Orlando, USA, 2003.

[3] R. W. Picard. Affective Computing. MIT Press, Massachusetts, USA, 1997.

[4] S.C. Marsella and J. Gratch. EMA: A process model of appraisal dynamics. Cognitive Systems Research, 10(1):70-90, 2009.

[5] J.A. Russell. Core affect and the psychological construction of emotion. Psychological review, 110(1):145-172, 2003.

[6] A. van der Heide and G. Trivino. A prototype of a machine speaking with emotions. In Proceedings of Congreso Español sobre Tecnologías y Lógica Fuzzy, pages 55 - 60, Huelva, Spain, 2010.

[7] A. van der Heide and G. Trivino. Simulating emotional personality in human computer interfaces. In IEEE World Congress Computational Intelligence, Barcelona, Spain, July 2010.

[8] K. Scherer, A. Schorr, and T. Johnstone, editors. Appraisal Processes in Emotion: Theory, Methods, Research. Oxford University Press, 2001.

[9] A. Ortony, G.L. Clore, and A. Collins. The cognitive structure of emotion. Cambridge University Press, Cambridge, UK, 1998.

[10] C. Becker, S. Kopp, and I. Wachsmuth. Simulating the emotion dynamics of a multimodal conversational agent. Affective Dialogue Systems, pages 154 - 165, 2004.

[11] M.S. El-Nasr, J. Yen, and Ioerger T.R. Flame fuzzy logic adaptive model of emotions. $A u$ tonomous Agents and Multi-Agent Systems, 3(3):219 - 257, 2000.

[12] J. Dias and A. Paiva. Feeling and reasoning: A computational model for emotional characters. Progress in Artificial Intelligence, pages 127 140, 2005.

[13] T.L. Gehm and K.R. Scherer. Factors determining the dimensions of subjective emotional space. In K.R. Scherer, editor, Facets of emo- 
tion. Lawrence Erlbaum Associates, Incorporated, Hillsdale, USA, 1988.

[14] J.A. Russell and A. Mehrabian. Evidence for a three-factor theory of emotions. Journal of Research in Personality, 11:273-294, 1977.

[15] A. Mehrabian. Analysis of the big-five personality factors in terms of the pad temperament model. Australian Journal of Psychology, 48(2):86-92, 1996.

[16] K.R. Scherer. Unconscious processes in emotion: The bulk of the iceberg. In P. Niedenthal, L.F. Barrett, and P. Winkielman, editors, The unconscious in emotion. Guilford Press, New York, USA, 2005.

[17] A. Ortony, D. Norman, and W. Revelle. Affect and Proto-Affect in Effective Functioning. In J. Fellous and M. Arbib, editors, Who needs emotions: The brain meets the machine. Oxford University Press, New York, USA, 2005.

[18] L.F. Barrett. Feeling Is Perceiving: Core Affect and Conceptualization in the Experience of Emotion. In L.F. Barrett, P.M. Niedenthal, and P. Winkielman, editors, Emotions: Conscious and Unconscious, pages 255-284. Guilford Press, 2005.

[19] J.A. Russell and L.F. Barrett. Core affect, prototypical emotional episodes, and other things called emotion: Dissecting the elephant. Journal of Personality and Social Psychology, 76(5):805-819, 1999.

[20] D. Lykken and A. Tellegen. Happiness is a stochastic phenomenon. Psychological Science, 7(3):186-189, 1996.

[21] J.P. Forgas. Mood and judgment: The affect infusion model (AIM). Psychological Bulletin, 117(1):39-66, 1995.

[22] R.W. Levenson. Emotion and the autonomic nervous system: A prospectus for research on autonomic specificity. In H.L. Wagner, editor, Social Psychophysiology and Emotion: Theory and clinical applications, pages 17-42. John Wiley \& Sons, New York, USA, 1988.

[23] P.J. Lang. The emotion probe. Studies of motivation and attention. The American Psychologist, 50(5):372, 1995.

[24] N. Schwarz. Feelings as information: Informational and motivational functions of affective states. In R.M. Sorrentino and E.T. Higgins, editors, Handbook of motivation and cognition: Foundations of social behavior. Guilford Press, New York, USA, 1990.

[25] R. Cowie, E. Douglas-Cowie, N. Tsapatsoulis, G. Votsis, S. Kollias, and W. Fellenz. Emotion recognition in human-computer interaction. IEEE Signal Processing Magazine, 1:32 $-80,2001$.

[26] S. Marsella, L Johnson, and C. LaBore. Interactive pedagogical drama. In Proceedings of the Fourth International Conference on Autonomous Agents, pages 301-308, 2000.
[27] S. Marsella and J. Gratch. Modeling coping behavior in virtual humans: don't worry, be happy. In Proceedings of the second international joint conference on Autonomous agents and multiagent systems, Melbourne, Australia, 2003. ACM.

[28] L.A. Zadeh. The concept of a linguistic variable and its application to approximate reasoning i, ii, iii. Information Sciences, 1975.

[29] L.A. Zadeh. Fuzzy sets, fuzzy logic, and fuzzy systems: selected papers by Lotfi A. Zadeh. World Scientific Publishing Co Pte Ltd, Singapore, Singapore, 1996.

[30] L.A. Zadeh. Fuzzy logic = computing with words. IEEE Transactions on Fuzzy Systems, 4(2):103-111, 1996.

[31] R.R. Yager and L.A. Zadeh, editors. An Introduction to Fuzzy Logic Applications in Intelligent Systems. Kluwer Academic Publishers, Norwell, USA, 1992.

[32] G. Trivino, A. Alvarez-Alvarez, and G. Bailador. Application of the computational theory of perceptions to human gait pattern recognition. Pattern Recognition, 43(7):2572-2581, 2010.

[33] A. Alvarez and G. Trivino. Comprehensible model of a quasi-periodic signal. In Proceedings of the 9th International Conference on Intelligent Systems Design and Applications, 2009.

[34] G. Trivino and A. van der Heide. An experiment on the description of sequences of fuzzy perceptions. In Proceedings of the International Conference on Hybrid Intelligent Systems, Barcelona, Spain, 2008.

[35] G. Trivino and A. van der Heide. Linguistic summarization of the human activity using skin conductivity and accelerometers. In Proceedings of the Conference Information Processing and Management of Uncertainty in Knowledge Based Systems, Malaga, Spain, 2008.

[36] R.R. McCrae and O.P. John. An introduction to the five-factor model and its applications. Journal of personality, 60(2):175-215, 1992.

[37] F. Dzogang, M.J. Lesot, M. Rifqi, and B. Bouchon-Meunier. Expressions of graduality for sentiments analysis - a survey. In Proceedings of fuzzIEEE 2010 (WCCI'2010), pages 1394 - 1400, 2010.

[38] P. Ekman and W.V. Friesen. Facial Action Coding System: A Technique for the Measurement of Facial Movement. Consulting Psychologists Press, Palo Alto, 1978.

[39] P. Ekman and E.L. Rosenberg. What the Face Reveals: Basic and Applied Studies of Spontaneous Expression Using the Facial Action Coding System. Oxford University Press, USA, 2005 . 